\title{
Clinical features, acute complications, and outcome of Salmonella meningitis in children under one year of age in Taiwan
}

\author{
Hung-Ming Wu ${ }^{1,7,8^{*}}$, Wan-Yu Huang ${ }^{5}$, Meng-Luen Lee ${ }^{2}$, Albert D Yang ${ }^{3}$, Ko-Ping Chaou ${ }^{4}$, Lin-Yu Hsieh ${ }^{6}$
}

\begin{abstract}
Background: Salmonella meningitis remains a threat to children below two years of age in both developing and developed countries. However, information on such infections has not been well characterized. We analyzed data related to twelve years of experience in order to clarify the comprehensive features of Salmonella meningitis in our patients, including admission characteristics, acute complications, and long-term outcome.

Methods: The records of patients with spontaneous Salmonella meningitis from 1982 to 1994 were retrospectively reviewed. The long-term outcome was prospectively determined for survivors at school age by the developmental milestones reported by their parents and detailed neurological evaluation along with intelligence, hearing, visual, speech and language assessments.

Results: Of the twenty-four patients, seizures were noted in fifteen (63\%) before admission and thirteen (54\%) during hospitalization. Acute complications mainly included hydrocephalus (50\%), subdural collection (42\%), cerebral infarction (33\%), ventriculitis (25\%), empyema (13\%), intracranial abscess (8\%), and cranial nerve palsy (8\%). Three patients (13\%) died during the acute phase of Salmonella meningitis. The twenty-one survivors, on whom we followed up at school age, have sequelae consisting of language disorder (52\%), motor disability (48\%), intelligence quotient < $80(43 \%)$, epilepsy (33\%), sensorineural hearing loss (17\%), visual deficits (10\%), abducens nerve palsy (5\%), microcephaly (5\%), and hydrocephalus (5\%). Overall, good outcome was noted in six (28.6\%) of twenty-one survivors, mild sequelae in three (14.2\%), moderate in six (28.6\%), and severe in six (28.6\%).

Conclusion: Salmonella meningitis in neonates and infants had a wide spectrum of morbidity and acute complications, leading to a complicated hospital course and subsequently a high prevalence of permanent adverse outcome. Thus, early recognition of acute complications of Salmonella meningitis and a follow-up plan for early developmental assessment of survivors are vital.
\end{abstract}

\section{Background}

Salmonella strains are an important pathogen of childhood bacterial meningitis in many developing countries, accounting for $5-13 \%$ of acute bacterial meningitis in young children in the 1980s-2000s [1-4]. In developed countries, Salmonella meningitis is a rare form (1\% or less) of acute bacterial meningitis, but such infections are serious and thus still a threat to children below the age of two [5,6]. In 1907, Ghon reported the first case of Salmonella meningitis [7]. The previous reports suggested that

\footnotetext{
* Correspondence: whmhwy@gmail.com

'Department of Neurology, Changhua Christian Hospital, Changhua, Taiwan Full list of author information is available at the end of the article
}

Salmonella meningitis was associated with a very high prevalence (50-90\%) of morbidity, presenting variable complications, and a high mortality rate of up to $50-70 \%$ [8-15]. However, comprehensive information regarding Salmonella meningitis, including clinical features, complications, and long-term sequelae among survivors has not been well characterized. To clarify these systemic features, particularly long-term outcomes of Salmonella meningitis occurring in neonates and infants primarily admitted to Changhua Christian Hospital from 1982 to 1994, the clinical characteristics of the disease present at hospital admission were retrospectively reviewed, and a follow-up assessment of patients at school age was prospectively undertaken.

\section{C) Biomed Central}




\section{Methods}

Changhua Christian Hospital is a teaching hospital in central Taiwan that serves as a referral center as well as a primary care facility. From 1982 to 1994, we collected one to three cases with Salmonella meningitis annually for a total of twenty six patients. Twenty four of them received first aid and all medical care in our hospital, and the other two were referred to us after partial antibiotic treatment by local medical care. After 1994, only a few cases with Salmonella meningitis were collected in our hospital due to public health improvements in Taiwan, and they were referred by local medical care. Since the group of referred cases with Salmonella meningitis was small and treated with varied durations of antibiotics before admission, this group was excluded from our study. The medical records of all patients primarily admitted to our hospital from 1982 to 1994 with spontaneous Salmonella meningitis were reviewed. They fulfilled the entry criteria for diagnosis by the presence of Salmonella species in cerebrospinal fluid (CSF) culture, which further supported infection based on pleocytosis $\left(>30 / \mathrm{mm}^{3}\right)$ with predominant neutrophilia and hypoglycorrhachia. Records were examined for initial clinical presentations, demographic features, laboratory data, acute complications at hospitalization, and antibiotic therapy. In this study, the modified Grady coma scale was used to measure the level of consciousness, classing children on a scale of I to $\mathrm{V}$ along a scale of lethargy or irritability (grade I), stupor (grade II), deep stupor (grade III), coma with abnormal posturing (grade IV), and coma without any response (grade V) [16]. Follow-up on surviving cases was performed at pediatric neurology outpatient clinics after discharge. This study was conducted with the approval of the Ethical Research Committee of Changhua Christian Hospital. Written informed consents were obtained from the participants and their parents after full oral and written explanation of the evaluation procedures, and the purpose of the study.

The survivors and/or their parents were asked to complete a questionnaire detailing developmental problems and presence of a seizure disorder. Developmental delay was defined as a child not reaching developmental milestones at the expected times, including motor development, hearing, speech and language. In addition, a pediatric neurologist determined the presence of motor disabilities and sequelae in survivors based on neurological examinations. Motor disability was defined as reduced ability to perform normal human motor functions, such as standing and walking. Hearing function was assessed with conventional behavioral pure-tone audiometry. General intelligence was measured using the Wechsler Intelligence Scale for Children-Revised
(WISC-R) [17]. Language ability was assessed with a language assessment test as previously described [18]. Cerebral background activity and epileptiform discharge were detected by electroencephalography (EEG). Cranial computed tomography (CT) scan was also performed to document brain tissue changes following Salmonella meningitis.

In addition to considering individual assessment results and events of Salmonella meningitis during hospitalization, the overall outcome was classified according to previous reports with a few modifications [19]. These were as follows: (1) death: death was associated with Salmonella meningitis; (2) severe adverse outcome: severe cerebral palsy (vegetative state and/or tetraparesis, tetraplegia), profound or severe intellectual impairment (intelligence quotient (IQ) score $<55$ ), profound or severe delayed language development ( $\geq 3$ standard deviations (SD) below the mean on the full score of the preschool language scale), intractable epilepsy, sensorineural hearing loss $\geq 60 \mathrm{~dB}$, and/or bilateral cranial nerve palsy or deficits; (3) moderate adverse outcome: moderate to mild cerebral palsy (hemiparesis or paraparesis), moderate intellectual impairment (IQ score between 55 and 69), moderate delayed language development ( $<3 \mathrm{SD}$ and $\geq 2$ SD below the mean), sensorineural hearing loss (59 to $40 \mathrm{~dB}$ ), one side of cranial nerve palsy or deficits, and/or epilepsy; (4) mild adverse outcome: minimal neurological impairment (generalized hyperreflexia and/or clumsiness), mild intellectual impairment (IQ score between 70 and 80), mild delayed language development $(<2 \mathrm{SD}$ and $\geq 1.5 \mathrm{SD}$ below the mean), and/or hydrocephalus without complications; and (5) good outcome: no motor disability, no intellectual impairment (IQ score > 80), and normal language development (<1.5 SD from the mean).

Data is expressed as mean \pm SD or median (range). Clinical features at the time of admission and acute complications of Salmonella meningitis were evaluated to find the relevant predictors associated with death, severe adverse outcome, and moderate adverse outcome using the 2-tailed Fisher's exact test (SPSS for Windows, version 15, SPSS Inc, Chicago, IL). $P$ value $<0.05$ was considered significant.

\section{Results}

\section{Clinical Characteristics and Acute Complications of Salmonella Meningitis}

We collected a total of twenty-four patients (six neonates and eighteen infants) with Salmonella meningitis, who had not received any treatment for the illness before coming to our hospital. All children with growth of Salmonella from CSF also fit the other criteria of the CSF pleocytosis $\left(>30 / \mathrm{mm}^{3}\right)$ with predominant neutrophilia and hypoglycorrhachia for meningitis. The records 
of all eligible patients presented for Salmonella meningitis were fully reviewed and all survivors were completed follow-up. Their basic data at the acute phase of Salmonella meningitis is summarized in Table 1. HIV infection and other risk factors for invasive disease were absent in our cases. They were admitted to our hospital within three days after symptom onset. The most common agents were Salmonella enteritidis Group D1 (41.7\%); others included Salmonella enteritidis Group B (12.5\%), Salmonella enteritidis Group C1 (8.3\%), Salmonella enteritidis Group C2 (4.2\%) and Salmonella species (33.3\%). Clinical features were similar in both the neonate and infant groups at the time of admission, except that a bulging anterior fontanel appeared to be more common in infants (61\%) than in neonates (33\%). Seizures were noted in 15 (63\%) of the twenty-four patients before admission. Seizures occurred in thirteen (54\%) during hospitalization, and ten (77\%) of those seizures were not well controlled even though antiepileptic drugs were administrated for $>48$ hours. Six (25\%) had no seizures noted in the course of Salmonella meningitis. The laboratory findings for the twenty-four patients are listed in Table 2. Cerebrospinal fluid (CSF)/blood glucose ratio $<0.5$ was $83 \%$ of those cases and CSF protein levels $>200 \mathrm{mg} / \mathrm{dl}$ in $54 \%$ of the patients.

Initial antibiotics that covered Salmonella species were used for the twenty-four patients, based on the results of gram stains and cultures of blood and CSF. They were treated with fourteen different antimicrobial regimens, and most received chloramphenicol or ampicillin in combination with one of the third generation cephalosporins (cefotaxime, moxalaxtam, ceftriaxone, and ceftazidime) (Table 1). In vitro sensitivities to the third generation cephalosporins, chloramphenicol, and ampicillin were $100 \%, 54.5 \%$, and $45.8 \%$, respectively. Antimicrobial therapy durations were $32.1 \pm 12.2$ days (range, 14 to 74 days). One case had acute complications, including refractory seizures, ventriculitis, and severe hydrocephalus, and received a ventriculoperitoneal shunt. This patient had a relapse of Salmonella meningitis with the same serotype of Salmonella 33 days after treatment with susceptible antibiotics for 56 days.

During hospitalization, brain sonography $(\mathrm{N}=22)$ and/or CT scan $(\mathrm{N}=18)$ and/or ventricular tap $(\mathrm{N}=$ 12) were performed to detect acute intracranial complications and to diagnose ventriculitis. Those complications included hydrocephalus (50\%), subdural collection $(42 \%)$, cerebral infarction (33\%), ventriculitis (25\%), empyema (13\%), intracranial abscess $(8 \%)$, and cranial nerve palsy $(8 \%)$ (Table 3$)$. Of twelve cases with hydrocephalus, six had ventriculitis, and three with severe non-communicating type received a ventriculoperitoneal shunt during hospitalization. Eight patients had cerebral infarction and most of them had one or more associated
Table 1 Admission features and progress of the twentyfour patients with Salmonella meningitis

\begin{tabular}{|c|c|}
\hline Characteristics & $\begin{array}{c}\text { Cases }(\mathrm{N}=24) \\
\mathrm{n}(\%)\end{array}$ \\
\hline Male: Female & 15: 9 \\
\hline Age at onset, days* & $\begin{array}{l}79 \pm 44.5 \\
\text { (range, } 5 \text { to } 266)\end{array}$ \\
\hline Onset of symptoms before admission, days* & $\begin{array}{c}2.1 \pm 0.74 \\
\text { (range, } 1 \text { to } 3 \text { ) }\end{array}$ \\
\hline \multicolumn{2}{|l|}{$\begin{array}{l}\text { Clinical features present between onset of } \\
\text { symptoms and day of diagnosis }\end{array}$} \\
\hline Fever & $24(100)$ \\
\hline Lethargy & $6(25)$ \\
\hline Irritability & $10(42)$ \\
\hline Poor feeding & $20(83)$ \\
\hline Diarrhea & $8(33)$ \\
\hline Vomiting & $9(38)$ \\
\hline Bulging anterior fontanel & $13(54)$ \\
\hline Nuchal rigidity & $9(38)$ \\
\hline Seizure & $15(63)$ \\
\hline \multicolumn{2}{|l|}{ Salmonella isolates } \\
\hline CSF & $24(100)$ \\
\hline Blood & $13(59)$ \\
\hline Stool & $3(17)$ \\
\hline Urine & $1(6)$ \\
\hline \multicolumn{2}{|l|}{ Antibiotics } \\
\hline $\mathrm{CM}$ & $1(4)$ \\
\hline$A C, C M$ & $2(8)$ \\
\hline AC, third-generation cephalosporins & $6(25)$ \\
\hline CM, third-generation cephalosporins & $4(17)$ \\
\hline$A C, C M$, third-generation cephalosporins & $2(8)$ \\
\hline AG, third-generation cephalosporins & $2(8)$ \\
\hline Third-generation cephalosporins & $7(29)$ \\
\hline Duration of hospitalization, days* & $\begin{array}{l}32.1 \pm 12.2 \\
\text { (range, } 14 \text { to } 74 \text { ) }\end{array}$ \\
\hline \multicolumn{2}{|l|}{ Condition at discharge } \\
\hline Survival & $21(88)$ \\
\hline No disability & $8(33)$ \\
\hline Disability & $13(54)$ \\
\hline Ongoing seizure & $7(29)$ \\
\hline Focal motor weakness & $10(42)$ \\
\hline Cranial nerve palsy & $2(8)$ \\
\hline Death & $3(13)$ \\
\hline Relapse & $1(4)$ \\
\hline
\end{tabular}

*Values are presented as mean \pm SD with range in parentheses; $\mathrm{n}(\%)$, case number with percentage of the patients with positive findings in parentheses; "Denotes CSF cultures done in 24 cases, blood cultures in 22, urine cultures in 16 , and stool cultures in 18; third-generation cephalosporins: cefotaxime, moxalaxtam, ceftriaxone, and ceftazidime; AC, Ampicllin; AG, aminoglycoside; $\mathrm{CM}$, Chloramphenicol; disability, impairment of physical ability and seizure.

and concomitant complications, mainly hydrocephalus (50\%), seizures (75\%), subdural effusion (40\%), empyema and brain abscess (25\%). Four children were found to have focal intracranial infection: two had subdural empyema, one had subdual empyema plus brain abscess, 
and one had multiple abscesses. All four patients also had other associated and concomitant complications such as prolonged seizures (100\%), cerebral infarction (50\%), ventriculitis (50\%), and abducens nerve palsy (25\%). They all received surgical management to drain brain abscess and empyema, and had severe neurological disability at discharge.

\section{Outcome of Patients with Salmonella Meningitis for School-Age Survivors}

Overall, good outcome was observed in six (28.6\%) of twenty-one survivors, mild adverse outcome in three $(14.2 \%)$, moderate in six $(28.6 \%)$, and severe in six (28.6\%). Table 3 summarizes the relevant unfavorable factors associated with outcome. There was a higher prevalence of acute complications and decreased level of consciousness in children who had poor outcome than in those with mild adverse and good outcomes.

Three (13\%) of the twenty-four patients with Salmonella meningitis died during hospitalization. The remaining twenty-one survivors were prospectively followed up when they were at school age (median 9.4, range 7.8 to 12.2 years) and then evaluated (Table 4 ). Developmental delay occurred in fourteen (67\%) cases. Nearly half $(48 \%)$ of the survivors had motor disabilities. Among five patients who had severe disabilities, two had spastic tetraplegia with/without urine and stool incontinence, and three had spastic tetraplegia in a vegetative state. Among four with moderate impairment, three had spastic hemiparesis and one had spastic paraparesis. One patient with mild impairment showed slight clumsiness and generalized hyperreflexia. In addition, ataxia was noticed in two patients with hemiparesis, and involuntary movement was noticed in three patients with tetraplegia.

Table 2 Analysis of peripheral leukocytes and CSF at the time of diagnosis

\begin{tabular}{|c|c|}
\hline Findings & Cases $(\mathrm{N}=24) \mathrm{n}(\%)$ \\
\hline \multicolumn{2}{|l|}{ Peripheral blood data } \\
\hline Total WBC/ $\left.\mu\right|^{*}$ & $12210(1570-28900)$ \\
\hline Segmented $\mathrm{WBC} /\left.\mu\right|^{*}$ & $4760(527-17138)$ \\
\hline Banded $\mathrm{WBC} / \mu \mathrm{I}^{*}$ & $389(0-4624)$ \\
\hline Platelet $\left(\times 10^{4} / \mu \mathrm{l}\right)^{*}$ & $32.7(7.4-87.5)$ \\
\hline \multicolumn{2}{|l|}{ CSF data } \\
\hline Total WBC/ $\left.\mu\right|^{*}$ & $1852(68-30600)$ \\
\hline $\mathrm{PMN} / \mu \mathrm{l}^{*}$ & $1490(65-24480)$ \\
\hline Glucose $(\mathrm{mg} / \mathrm{L})^{*}$ & $15(0-69)$ \\
\hline Protein $(\mathrm{mg} / \mathrm{dll})^{*}$ & $330(40-740)$ \\
\hline CSF/blood glucose ratio $<0.5$ & $20(83)$ \\
\hline CSF protein $>200$ mg/dl & $13(54)$ \\
\hline Gram stain positive & $24(100)$ \\
\hline
\end{tabular}

* Values are presented as median (complete range); WBC, white blood cell; PMNs, polymorphonuclear leukocytes.
Seven (33\%) cases of the twenty-one survivors developed epilepsy. Five had refractory epilepsy that developed at the time of their acute illness, and the other two developed epilepsy post Salmonella meningitis at two and four years. All seven cases had focal or multifocal epileptiform discharges on EEG recordings. In addition, one survivor who did not display clinical symptoms of seizure had abnormal focal epileptiform discharges.

In sixteen $(76 \%)$ of twenty-one children, brain CT scans revealed abnormal findings, suggesting old infarction and lesions associated with acute Salmonella meningitis. Moderate hydrocephalus was detected in one child, who had generalized tendon hyperreflex and good intelligence. Another patient who had received a ventriculoperitoneal shunt at age six months due to severe hydrocephalus had a normal sized ventricle.

One (5\%) of twenty-one children had had left abducens nerve palsy since Salmonella meningitis and two (10\%) had visual deficits. Sensorineural hearing impairment was noticed in three (17\%) of the eighteen survivors tested by pure-tone audiometry. Two had severe unilateral sensorineural loss (>60 dB) while one had mild unilateral sensorineural loss $(40 \mathrm{~dB})$. The other three survivors could not be tested due to their severe disabilities.

The language assessment test for school-age children was designed to identify language comprehension and verbal expression ability [18]. Of the twenty-one survivors who completed the test, eleven (52\%) were found to have speech and language developmental delay, of whom five had severe communication problems or absence of speech. Thus, these five could not perform the neuropsychological tasks on the WISC-R, and the estimated score for each was assigned a poor performance under -3 SD for age because of their severe intellectual disabilities. The other sixteen children completed the WISC-R. The median full-scale IQ for these 16 cases was 93.5 (range, 48 to 107). Two children had borderline learning difficulty and another two had a moderate learning difficulty or worse.

Table 3 summarizes the relevant predictors associated with death and severe to moderate adverse outcomes found by univariate analyses. The major unfavorable factors presented during Salmonella meningitis were conscious change, seizure during hospitalization, CSF/blood glucose ratio $<0.5$, CSF protein $>200 \mathrm{mg} / \mathrm{dl}$, focal intracranial infection, ventriculitis, and cerebral infarction. The features including seizure onset before admission, subdural collection, and hydrocephalus were not significantly associated with a poor long-term outcome.

\section{Discussion}

In this study, three out of twenty-four patients with spontaneous Salmonella meningitis died, representing a 
Table 3 The association of clinic features, CSF findings, and acute complications with the outcome in twenty-four children with Salmonella meningitis

\begin{tabular}{|c|c|c|}
\hline Variable & $\begin{array}{l}\text { Death, moderate and severe sequelae group } \\
\qquad(\mathrm{N}=15) \mathrm{n}(\%)\end{array}$ & $\begin{array}{l}\text { Mild sequelae and good outcome group } \\
\qquad(\mathrm{N}=9) \mathrm{n}(\%)\end{array}$ \\
\hline \multicolumn{3}{|l|}{ Level of consciousness } \\
\hline $\begin{array}{l}\text { Lethargy or irritability at } \\
\text { admission* }\end{array}$ & $13(87)$ & $3(33)$ \\
\hline Coma in hospital ${ }^{\# *}$ & $10(67)$ & $0(0)$ \\
\hline \multicolumn{3}{|l|}{ Seizures } \\
\hline Seizure before admission & $10(67)$ & $5(56)$ \\
\hline Seizure in hospital* & $12(80)$ & $1(11)$ \\
\hline \multicolumn{3}{|l|}{ Laboratory tests } \\
\hline CSF: blood glucose $<0.5^{*}$ & $15(100)$ & $5(56)$ \\
\hline CSF protein $>200 \mathrm{mg} / \mathrm{dl}^{*}$ & $11(73)$ & $2(22)$ \\
\hline \multicolumn{3}{|l|}{ Intracranial complications } \\
\hline Subdural collection & $6(40)$ & $4(44)$ \\
\hline Intracranial focal infection* & $5(33)$ & $0(0)$ \\
\hline Empyema & $3(20)$ & $0(0)$ \\
\hline Brain abscess & $2(13)$ & $0(0)$ \\
\hline Cerebral infarction* & $8(53)$ & $0(0)$ \\
\hline Ventriculitis* & $6(40)$ & $0(0)$ \\
\hline Hydrocephalus & $9(60)$ & $3(33)$ \\
\hline
\end{tabular}

*Denotes a significant difference between death, moderate and severe sequelae group and mild adverse and good outcome group in the univariate analysis using Fischer's exact test $(p<0.05)$; ${ }^{*}$ Denotes Grady coma scale IV and V.

Table 4 Long-term outcome associated with acute main complications for Salmonella meningitis in twenty-one survivors

\begin{tabular}{|c|c|c|c|c|c|c|c|c|}
\hline \multirow[t]{2}{*}{ Findings } & \multirow{2}{*}{$\begin{array}{l}\text { Survivors }(\mathrm{N}=21) \\
\mathrm{n} / \text { total }(\%)^{*}\end{array}$} & \multicolumn{7}{|c|}{ Number of acute main complications in each particular outcome } \\
\hline & & Seizure ${ }^{\#}$ & $\begin{array}{l}\text { Sudural } \\
\text { effusion }\end{array}$ & Hydro- cephalus & Ventr-iculitis & Em-pyema & $\begin{array}{l}\text { Brain } \\
\text { abcess }\end{array}$ & $\begin{array}{l}\text { Cerebral } \\
\text { infarction }\end{array}$ \\
\hline \multicolumn{9}{|l|}{ Development } \\
\hline Delayed & $14 / 21(67)$ & 11 & 5 & 8 & 5 & 3 & 2 & 7 \\
\hline Normal & $7 / 21(23)$ & 0 & 4 & 3 & 0 & 0 & 0 & 0 \\
\hline \multicolumn{9}{|l|}{$\begin{array}{l}\text { Motor } \\
\text { disability }\end{array}$} \\
\hline Severe & $5 / 21(24)$ & 5 & 2 & 5 & 5 & 1 & 2 & 3 \\
\hline Moderate & 4/21 (19) & 3 & 3 & 1 & 0 & 1 & 0 & 4 \\
\hline Mild & $1 / 21(5)$ & 0 & 0 & 0 & 0 & 0 & 0 & 0 \\
\hline Normal & $11 / 21(52)$ & 3 & 4 & 4 & 0 & 1 & 0 & 0 \\
\hline $\begin{array}{l}\text { Hearing } \\
\text { Problems }\end{array}$ & $3 / 18(17)$ & 2 & 1 & 3 & 1 & 1 & 1 & 0 \\
\hline Epilepsy & $7 / 21(33)$ & 6 & 2 & 4 & 4 & 3 & 2 & 5 \\
\hline Microcephaly & $1 / 21(5)$ & 1 & 0 & 1 & 1 & 0 & 1 & 1 \\
\hline Hydrocephalus & $1 / 21(5)$ & 0 & 0 & 0 & 0 & 0 & 0 & 0 \\
\hline Visual deficit & 2/21 (10) & 1 & 2 & 1 & 0 & 0 & 0 & 1 \\
\hline CN6 palsy & $1 / 21(5)$ & 0 & 1 & 1 & 0 & 0 & 0 & 0 \\
\hline \multicolumn{9}{|l|}{ Intelligence } \\
\hline $\mathrm{IQ}<55$ & 6/21 (29) & 6 & 2 & 5 & 5 & 2 & 2 & 4 \\
\hline IQ 55-69 & $1 / 21(5)$ & 1 & 0 & 0 & 0 & 1 & 0 & 0 \\
\hline IQ 70-80 & $2 / 21(10)$ & 1 & 1 & 1 & 0 & 0 & 0 & 1 \\
\hline $\mathrm{IQ}>80$ & $12 / 21(57)$ & 3 & 6 & 5 & 0 & 0 & 0 & 2 \\
\hline \multicolumn{9}{|l|}{$\begin{array}{l}\text { Speech/ } \\
\text { language }\end{array}$} \\
\hline Abnormal & $11 / 21(52)$ & 9 & 4 & 7 & 5 & 3 & 2 & 6 \\
\hline Normal & $10 / 21(48)$ & 2 & 5 & 4 & 0 & 0 & 0 & 1 \\
\hline
\end{tabular}

*Values are expressed as number of patients with each particular outcome/number of patients examined, and numbers in parentheses are percentages; ${ }^{\text {SSeizure }}$ in hospital; CN6, Abducens nerve palsy; MR, mental retardation; IQ, intelligence quotient. 
mortality rate of $13 \%$; eighteen (75\%) cases developed at least one complication at the acute phase of Salmonella meningitis, leading to a complicated clinical course. At school-age follow-up, fifteen (71\%) of the twenty-one survivors had subsequent motor disabilities, epilepsy, language delay, and intelligence impairment (Table 4), and most (58\%) suffered from moderate to severe sequelae. We further revealed that death and major adverse outcomes were highly associated with seven unfavorable factors during Salmonella meningitis: conscious change, seizure during hospitalization, CSF/blood glucose ratio $<0.5$, CSF protein $>200 \mathrm{mg} / \mathrm{dl}$, focal intracranial infection, ventriculitis, and cerebral infarction (Table 3)

The incidence (71\%) of mild to severe adverse long-term outcomes in our twenty-one survivors was high, compared with those in the previous studies [8-13,15]. One explanation is that our cases demonstrated more complicated involvement of the brain, such as cerebral infarction. On the other hand, our children were followed up at school age. Almost all previous studies involved follow-up of children aged less than five years [8-13,15]. Since survivors remain at high risk for neurologic sequelae and lifelong impairment following meningitis [20,21], there is difficulty in assessing those cases, particularly during the neonate period or during the first two years of life. Our study may thus provide a more accurate assessment for survivors, and give an incidence of adverse long-term outcome resulting from Salmonella meningitis.

Previous studies have demonstrated many prognostic factors for an unfavorable outcome of bacterial meningitis in children, such as Streptococcus pneumoniae meningitis [2,22]. Since Salmonella meningitis is a relatively rare form of bacterial meningitis in neonates and infants, until now, there have been few studies that focus on the predictive factors for long-term outcomes $[10,12]$. Although our case number was not so large, this study does allow us to assess trends in the distribution of an adverse outcome in relation to factors discernible at the acute phase of Salmonella meningitis (Table 3). The group with major adverse outcome and death was highly associated with seven unfavorable factors in comparison to the group with mild to no adverse outcome in univariate analysis $(p<0.05$, Fisher's exact test), e.g. conscious alteration, late seizures, high CSF protein, low CSF/blood glucose ratio, cerebrovascular involvement, and intracranial infectious complications (Table 3). These factors are consistent with most predictive factors found in other bacterial meningitis [2,22].

Treatment of Salmonella meningitis has not been well defined. Antimicrobial agents, including chloramphenicol, ampicillin, and cotrimoxazole, had a low cure rate $(\sim$ $40 \%)$ and a highly associated mortality ( 45\%) [23]. Third-generation cephalosporins and fluoroquinolones had a high cure rate (> 80\%) and lowered the associated mortality $(<10 \%)$ [10,24-26]. Thus, those two antibiotics would be regarded as the optimal antimicrobial regimens in the treatment of Salmonella meningitis [26]. All our patients were treated initially with antibiotics to which their Salmonella species was susceptible, and most received third-generation cephalosporins, combined with chloramphenicol or ampicillin. Similar to the previous report [10], the mortality rate in our cases declined to about $10 \%$, but the morbidities remained high, up to $75 \%$. The results suggest that susceptible antibiotics for treatment of Salmonella meningitis in children were unsatisfactory. Adjunctive dexamethasone, therefore, may be recommended to attenuate the effects of the acute innate inflammatory response to bacterial invasion within the CNS $[27,28]$, although the benefits of steroids in meningitis seemed controversial in developing countries [29]. In addition, the patient should be monitored in the intensive care unit for any morbidity, such as seizure, in order to detect its potential complication.

In the present study, there are some limitations that merit discussion. The admission features of our patients were retrospectively collected, and the sample size was not large enough for a good statistical analysis. The assessment methods were not fully validated. For instance, a standard clinical examination was performed to identify children with a gross motor impairment, which might not fully assess motor function in those with minimal deficits. Nevertheless, about a half of our postmeningitic children followed up at school age had neuromuscular disability, which was high in prevalence among bacterial meningitis [30-32]. Second, the study was limited by the lack of a sibling control group for comparison, since the possibility that some of the cognitive impairment detected might not be attributive to Salmonella meningitis must be considered. However, each of the tests used for neuropsychological and language assessment had been validated for normal populations, providing assurance that the intellectual deficits noted were the result of Salmonella meningitis. Our children with moderate to profound MR had more risk factors $(5.2 \pm 0.5)$ than those with normal average IQ and mild MR $(1.6 \pm 1.1)$ (Table 3$)$. Thus, the results suggest that their motor disabilities and cognitive impairment might be highly related to the severity of their medical condition during the acute phase.

\section{Conclusions}

In summary, the present study provides more insight into a clinical spectrum and long-term outcome in the patients with Salmonella meningitis. Our study further revealed that Salmonella meningitis resulted in a high prevalence of long-term adverse outcome, being linked with its insult of acute complications, and a broad range of the potential neurological sequelae after meningitis 
remained with age. Early recognition of acute complications of Salmonella meningitis and a follow-up plan for early developmental assessment of survivors are vital.

\section{Abbreviations}

CSF: cerebrospinal fluid; EEG: electroencephalography; IQ: intelligence quotient; WISC-R: Wechsler Intelligence Scale for Children-Revised.

\section{Acknowledgements}

This was supported by the Grant 83-CCH-IRP-01 from Changhua Christian Hospital for Medical Research. We thank the children and their parents who participated in this study, Rorn-Ting Huang who assisted in the children's follow up, and Chiu-Yueh Yang who analyzed the data. We are grateful to Professor Ching-Shiang Chi and Dr Man-Chi Lo for constructively reviewing the manuscript.

\section{Author details}

'Department of Neurology, Changhua Christian Hospital, Changhua, Taiwan. ${ }^{2}$ Pediatrics, Changhua Christian Hospital, Changhua, Taiwan. ${ }^{3}$ Neuroradiology, Changhua Christian Hospital, Changhua, Taiwan. ${ }^{4}$ Psychiatry, Changhua Christian Hospital, Changhua, Taiwan. ${ }^{5}$ Department of Neuropediatrics, Kuang-Tein General Hospital, Taichung, Taiwan. ${ }^{6}$ Research center of speech and language disorders, Department of Pediatrics, Changhua Christian Hospital, Changhua, Taiwan. ${ }^{7}$ Neuroscience \& Psychiatry Research Lab, Changhua Christian Hospital, Changhua, Taiwan. ${ }^{8}$ Graduate Institute of Acupuncture Science, China Medical University, Taichung, Taiwan.

\section{Authors' contributions}

HMW was responsible for the design, the concept and the writing of this manuscript. WYH and MLL contributed to data collection and conducted the primary analysis. ADY conducted image analysis. KPC contributed to speech and language assessment and its analysis. LYH contributed to neuropsychological assessment and its analysis. All authors read and approved the manuscript.

\section{Competing interests}

The authors declare that they have no competing interests.

Received: 2 June 2010 Accepted: 27 January 2011

Published: 27 January 2011

\section{References}

1. Salaun-Saraux P, Saraux A, Lepage P, Van GC, Hitimana DG, Bazubagira A, Cénac A, Bogaerts J: Septic meningitis in children in Rwanda from 1983 to 1990. Retrospective study at the Kigali Hospital Center. Med Trop 1995, 55(1):41-45.

2. Molyneux EM, Walsh AL, Malenga G, Rogerson S, Molyneux ME: Salmonella meningitis in children in Blantyre, Malawi, 1996-1999. Ann Trop Paediatr 2000, 20(1):41-44.

3. Molyneux EM, Walsh AL, Forsyth H, Tembo M, Mwenechanya J, Kayira K, Bwanaisa L, Njobvu A, Rogerson S, Malenga G: Dexamethasone treatment in childhood bacterial meningitis in Malawi: a randomized controlled trial. Lancet 2002, 360(9328):211-218.

4. Chotpitayasunondh T: Bacterial meningitis in children: etiology and clinical features, an 11-year review of 618 cases. Southeast Asian J Trop Med Public Health 1994, 25(1):107-115.

5. Synnott MB, Morse DL, Hall SM: Neonatal meningitis in England and Wales: a review of routine national data. Archives of Disease in Childhood 1994, 71(2):f75-f80.

6. Klinger G, Chin CN, Beyene J, Perlman M: Predicting the outcome of neonatal bacterial meningitis. Pediatrics 2000, 106(3):477-482.

7. Ghon J: Bericht über denXIV: Internationalen Kongress für Hygience und Demographie. Berlin 1907, 4:21-23.

8. Kavaliotis J, Tsiaousi A, Papavasiliou D, Kansouzidou A: Non-typhoid Salmonella meningitis. Scand J Infect Dis 1994, 26(4):403-405.

9. Messer RD, Warnock TH, Heazlewood RJ, Hanna JN: Salmonella meningitis in children in far north Queensland. J Paediatr Child Health 1997, 33(6):535-538.
10. Huang LT, Ko SF, Lui CC: Salmonella meningitis: clinical experience of third-generation cephalosporins. Acta Paediatr 1997, 86(10):1056-1058.

11. Visudhiphan $P$, Chiemchanya $S$, Visutibhan A: Salmonella meningitis in Thai infants: clinical case reports. Trans R Soc Trop Med Hyg 1998, 92(2):181-184

12. Lee WS, Puthucheary SD, Omar A: Salmonella meningitis and its complications in infants. J Paediatr Child Health 1999, 35(4):379-382.

13. Sirinavin S, Chiemchanya S, Vorachit M: Systemic nontyphoidal Salmonella infection in normal infants in Thailand. Pediatr Infect Dis J 2001, 20(6):581-587.

14. Sangaré L, Kienou M, Lompo P, Ouédraogo-Traoré R, Sanou I, Thiombiano R, Lompo M, Diabaté A, Yaméogo S, Sanogo I, Guira C: Salmonella meningitis in Ouagadougou, Burkina Faso, from 2000 to 2004. Bull Soc Pathol Exot 2007, 100(1):53-56.

15. Molyneux EM, Mankhambo LA, Phiri A, Graham SM, Forsyth H, Phiri A, Walsh AL, Wilson LK, Molyneux ME: The outcome of non-typhoidal Salmonella meningitis in Malawian children 1 997-2006. Ann Trop Paediatr 2009, 29(1):13-22.

16. Cooper PR, Moody S, Clark WK, Kirkpatrick J, Maravilla K, Gould AL, Drane W: Dexamethasone and severe head injury. A prospective double-blind study. J Neurosurg 1979, 51(3):307-316.

17. Wechsler D: Wechsler Intelligence Score for Children-Revised. New York: Psychological Corporation. 1974.

18. Lin BG, Lin MS: J Hearing Language (Taiwan). 1994, 10:30-52, (In Chinese).

19. Mclntyre $P$, Jepson R, Leeder S, Irwig L: The outcome of childhood Haemophilus influenzae meningitis. A population based study. Med J Aust 1993, 159(11-12):766-772.

20. Grimwood K, Anderson P, Anderson V, Tan L, Nolan T: Twelve year outcomes following bacterial meningitis: further evidence for persisting effects. Arch Dis Child 2000, 83(2):111-116.

21. de Louvois J, Halket S, Harvey D: Neonatal meningitis in England and Wales: sequelae at 5 years of age. Eur J Pediatr 2005, 164(12):730-734.

22. Østergaard C, Konradsen HB, Samuelsson S: Clinical presentation and prognostic factors of Streptococcus pneumoniae meningitis according to the focus of infection. BMC Infect Dis 2005, 5:93.

23. Owusu-Ofori A, Scheld WM: Treatment of Salmonella meningitis: two case reports and a review of the literature. Int J Infect Dis 2003, 7(1):53-60.

24. Visudhiphan $P$, Chiemchanya S, Visutibhan A: Salmonella meningitis in Thai infants: clinical case reports. Trans R Soc Trop Med Hyg 1998, 92(2):181-184.

25. Schaad UB, abdus Salam M, Aujard Y, Dagan R, Green SD, Peltola H, Rubio TT, Smith AL, Adam D: Use of fluoroquinolones in paediatrics: consensus report of an International Society of Chemotherapy commission. Pediatr Infect Dis J 1995, 14(1):1-9.

26. Price EH, De Louvois J, Workman MR: Antibiotics for Salmonella meningitis in children. J Antimicrob Chemother 2000, 46(5):653-655.

27. Quagliarello VJ, Scheld WM: Treatment of bacterial meninigitis. N Engl J Med 1997, 336:708-714.

28. Quagliarello V, Scheld WM: Infectious disease: do steroids benefit patients with bacterial meningitis? Nat Rev Neurol 2010, 6(10):529-30.

29. Molyneux EM, Walsh AL, Forsyth H, Tembo M, Mwenechanya J, Kayira K, Bwanaisa L, Njobvu A, Rogerson S, Malenga G: Dexamethasone treatment in childhood bacterial meningitis in Malawi: a randomised controlled trial. Lancet 2002, 360(9328):211-218.

30. Taylor HG, Mills EL, Ciampi A, du Berger R, Watters GV, Gold R, MacDonald N, Michaels RH: The sequelae of Haemophilus influenzae meningitis in school-age children. N Engl J Med 1990, 323(24):1657-1663.

31. Grimwood K, Anderson VA, Bond L, Catroppa C, Hore RL, Keir EH, Nolan T, Roberton DM: Adverse outcomes of bacterial meningitis in school-age survivors. Pediatrics 1995, 95(5):646-656

32. Baraff $\mathrm{L}$, Lee $\mathrm{SI}$, Schriger DL: Outcomes of bacterial meningitis in children: a meta-analysis. Pediatr Infect Dis J 1993, 12(5):389-394.

\section{Pre-publication history}

The pre-publication history for this paper can be accessed here: http://www.biomedcentral.com/1471-2334/11/30/prepub

\section{doi:10.1186/1471-2334-11-30}

Cite this article as: Wu et al: Clinical features, acute complications, and outcome of Salmonella meningitis in children under one year of age in Taiwan. BMC Infectious Diseases 2011 11:30. 